\title{
Overexpression of UCP1 in tobacco induces mitochondrial biogenesis and amplifies a broad stress response
}

Pedro Barreto', Vagner Katsumi Okura', Izabella Agostinho Pena Neshich'1, Ivan de Godoy Maia ${ }^{3}$ and Paulo Arruda ${ }^{1,2^{*}}$

\begin{abstract}
Background: Uncoupling protein one (UCP1) is a mitochondrial inner membrane protein capable of uncoupling the electrochemical gradient from adenosine-5'-triphosphate (ATP) synthesis, dissipating energy as heat. UCP1 plays a central role in nonshivering thermogenesis in the brown adipose tissue (BAT) of hibernating animals and small rodents. A UCP1 ortholog also occurs in plants, and aside from its role in uncoupling respiration from ATP synthesis, thereby wasting energy, it plays a beneficial role in the plant response to several abiotic stresses, possibly by decreasing the production of reactive oxygen species (ROS) and regulating cellular redox homeostasis. However, the molecular mechanisms by which UCP1 is associated with stress tolerance remain unknown.

Results: Here, we report that the overexpression of UCP1 increases mitochondrial biogenesis, increases the uncoupled respiration of isolated mitochondria, and decreases cellular ATP concentration. We observed that the overexpression of UCP1 alters mitochondrial bioenergetics and modulates mitochondrial-nuclear communication, inducing the upregulation of hundreds of nuclear- and mitochondrial-encoded mitochondrial proteins. Electron microscopy analysis showed that these metabolic changes were associated with alterations in mitochondrial number, area and morphology. Surprisingly, UCP1 overexpression also induces the upregulation of hundreds of stress-responsive genes, including some involved in the antioxidant defense system, such as superoxide dismutase (SOD), glutathione peroxidase (GPX) and glutathione-S-transferase (GST). As a consequence of the increased UCP1 activity and increased expression of oxidative stress-responsive genes, the UCP1-overexpressing plants showed reduced ROS accumulation. These beneficial metabolic effects may be responsible for the better performance of UCP1-overexpressing lines in low pH, high salt, high osmolarity, low temperature, and oxidative stress conditions.

Conclusions: Overexpression of UCP1 in the mitochondrial inner membrane induced increased uncoupling respiration, decreased ROS accumulation under abiotic stresses, and diminished cellular ATP content. These events may have triggered the expression of mitochondrial and stress-responsive genes in a coordinated manner. Because these metabolic alterations did not impair plant growth and development, UCP1 overexpression can potentially be used to create crops better adapted to abiotic stress conditions.
\end{abstract}

Keywords: UCP1, Mitochondria, Oxidative stress, Biogenesis, Plant, Stress response

\footnotetext{
*Correspondence: parruda@unicamp.br

${ }^{1}$ Centro de Biologia Molecular e Engenharia Genética, Universidade Estadual

de Campinas (UNICAMP), 13083-875 Campinas, SP, Brazil

${ }^{2}$ Departamento de Genética e Evolução, Instituto de Biologia, Universidade

Estadual de Campinas (UNICAMP), 13083-875 Campinas, SP, Brazil

Full list of author information is available at the end of the article
} 


\section{Background}

Mitochondrial uncoupling protein one (UCP1) is a nuclearencoded protein located in the mitochondrial inner membrane. In the presence of fatty acids, UCP1 uncouples the electrochemical gradient from adenosine-5'-triphosphate (ATP) synthesis, dissipating energy as heat [1]. Mammalian UCP1 has long been investigated in brown adipose tissue (BAT) for its role in thermogenesis and the regulation of reactive oxygen species (ROS) production [2-4].

UCP1 has also been found in plants [5]. Similar to its mammalian orthologs, plant UCP1 belongs to a multigenic family whose members are expressed in a time- and tissuedependent manner and in response to low temperature [6-8]. Plant UCPs have also been shown to be involved in thermogenesis regulation in skunk cabbage [9] and climacteric increases in respiration in fruits [10], but the widespread presence of this protein in eukaryotic organisms suggests that it may have other functions [8]. The overexpression of Arabidopsis thaliana UCP1 (AtUCP1) in tobacco plants resulted in increased tolerance to oxidative stress [11]. In addition, the tobacco plants overexpressing AtUCP1 exhibited faster germination under control and stressful conditions, improved performance under drought and salt stresses, and increased rates of photosynthesis [12]. The mechanism underlying this increased stress protection is generally associated with decreased ROS production [11,12], but a recent study performed on Solanum lycopersicum (tomato) plants overexpressing a UCP gene suggest a wider role for UCPs' protective mechanisms by altering cell redox homeostasis and antioxidant capacity [13]. These previous studies regarding UCP overexpression, along with a study of an insertional knockout of AtUCP1 [14], suggest that UCPs may alter metabolism more globally, modulating mitochondrial, chloroplastic and cytosolic metabolism.

The role of mitochondria in energy metabolism and the stress response implies that this organelle communicates with other cellular compartments. Alteration of mitochondrial function modulates the expression of UCP1 and other nuclear-encoded mitochondrial proteins, including alternative oxidase (AOx) and type II NAD(P)H dehydrogenase, through mitochondria-to-nucleus signaling $[15,16]$. Mitochondria-to-nucleus communication has been extensively investigated in mammalian models, in which a signaling process coordinates the expression of genes encoded by the mitochondrial and nuclear genomes through mechanisms known as anterograde (nucleus to organelle) and retrograde (organelle to nucleus) signals [17]. The cytosolic concentrations of $\mathrm{Ca}^{2+}$ and ATP, together with the mitochondrial fission/fusion dynamics and ROS production, play central roles in the antero/retrograde signaling pathways and in the regulation of mitochondrial proliferation [18]. Both anterograde and retrograde signaling have been implicated in mitochondrial biogenesis
$[19,20]$, a process that is well documented in mammalian models [4] but poorly understood in plants [21]. In plants, the upregulation of nuclear-encoded mitochondrial genes have been seen during inflorescence development, especially during flowering [22-24], but the molecular components involved in the regulation of this process remains elusive. Recently, it has been shown that the pentatricopeptide proteins [25] and the transcription factors bZIP [26], WRKY [27], TCP [28] and NAC [29] may contribute to the retrograde regulation of mitochondrial/nucleus communication in plants.

In the present work, we investigated the mechanism of UCP1 action in the stress response. We used molecular, cellular and genomic tools to investigate the molecular and cellular events resulting from the overexpression of AtUCP1 in transgenic tobacco plants. We show that $A t U C P 1$ overexpression increases uncoupled respiration, decreases cellular ATP concentration, alters mitochondrial morphology, and triggers retrograde signaling, activating the expression of mitochondrial- and nuclear-encoded mitochondrial proteins. These changes are accompanied by a broad induction of stress-responsive genes that may help the AtUCP1-overexpressing cells reduce the levels of ROS and perform better under stress conditions. The results are discussed in the context of the link between mitochondrial biogenesis and the stress response in plants.

\section{Results}

Overexpression of the Arabidopsis UCP1 (AtUCP1) in tobacco shows no phenotypic alterations independent of its transgene expression levels

The AtUCP1 gene, encoding the Arabidopsis ortholog of mammalian UCP1 [6], was cloned under the control of the $35 \mathrm{~S}$ CaMV promoter and transformed into tobacco plants [11]. Two transgenic events, P49 and P07, which presented intermediary and high AtUCP1 expression, respectively, were chosen for this study. Although UCP1 acts directly in the mitochondrial respiratory chain by uncoupling the electron transport from ATP synthesis, no apparent phenotypic alterations were observed in the P49 and P07 transgenic lines compared with the wildtype (WT) plants (Figure 1A). This result diverges from previous findings showing increased shoot dry mass in plants overexpressing the same UCP1 [12], but it is consistent with observations of tomato plants overexpressing a UCP, which did not exhibit growth stimulation [13]. The addition of sucrose to the growth medium may have altered mitochondrial metabolism and biogenesis [24]. In the data presented in Figure 1A, we did not add sucrose to the nutrient solution.

The transgenic lines P49 and P07 presented 2- to 8-fold increases in AtUCP1 expression compared to WT plants in the two leaves sampled (Figure 1B). Immunoblotting using an anti-AtUCP1 polyclonal antibody, which recognizes the 

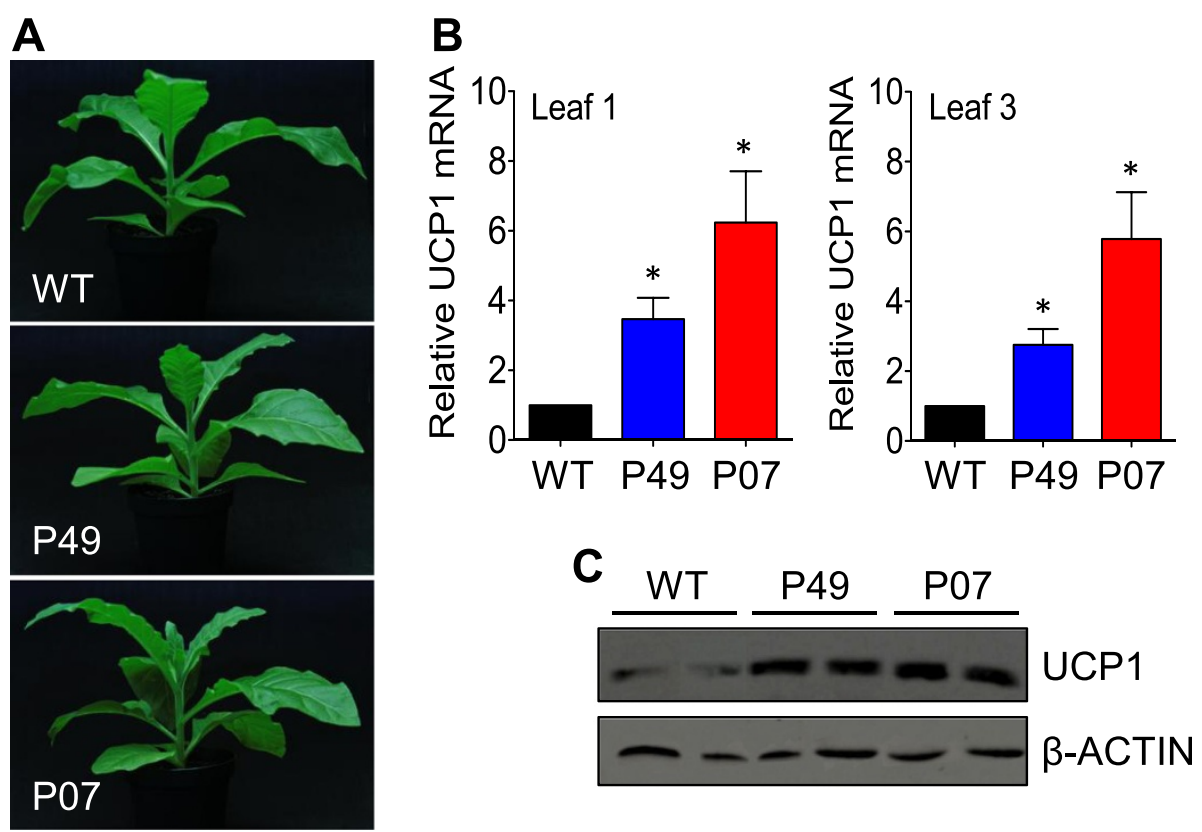

Figure 1 Transgenic tobacco overexpressing AtUCP1 exhibits a normal plant phenotype independent of the UCP1 expression level.

(A) Wild-type (WT) and AtUCP1 transgenic lines P49 and P07 grown for 12 weeks in 50:50 soil/vermiculite mixtures fertilized with half-strength MS medium not supplemented with sucrose. (B) Transcript abundance of AtUCP1 in L1 and L3 leaves of WT and transgenic lines P49 and P07. (C) Immunoblot analysis of $\mathrm{L} 3$ leaves of WT and transgenic lines P49 and P07 using an anti-AtUCP1 antibody. The loading control was examined using an anti- $\beta$-actin antibody. Panel (A) is representative of an experiment using 12 plants for each genotype $(n=12)$. Panel $(\mathbf{B})$ is representative of an experiment with 6 plants for each genotype $(n=6)$. Panel $(\mathbf{C})$ is representative of an experiment conducted in triplicate. ${ }^{*} p<0.05$ compared with the control. Error bars, mean \pm s.e.m.

tobacco and Arabidopsis UCP1, showed that the AtUCP1 protein (approximately $32 \mathrm{kD}$ ) was weakly detectable in WT leaves but accumulated at high levels in P49 and P07 transgenic lines (Figure 1C).

\section{AtUCP1 overexpression triggers mitochondria biogenesis through retrograde signaling}

Young and mature leaves differ in their responses to cold stress and photosynthetic activity [30,31]. Therefore, we examined whether young or more expanded leaves would respond differently to AtUCP1 overexpression. P07, P49 and WT plants were grown for 12 weeks, and apical leaves varying from youngest (L1) to oldest (L5) (Figure 2A) were sampled. Mesophyll protoplasts were prepared from L3, stained with the mitochondria-selective probe MitoTracker Red CM-H2XRos (Invitrogen, Carlsbad, CA, USA) and analyzed with fluorescent confocal microscopy. Both P49 and P07 transgenic lines showed 1.5- to 2-fold increases in the MitoTracker Red fluorescence signal compared with WT, indicating that UCP1 overexpression led to an increase in the mitochondrial number and/or volume (Figure 2B, C, and D). The Arabidopsis mitochondrial DNA (mtDNA) encodes 57 proteins involved in mitochondrial metabolism and mtDNA maintenance [32]. In mammals, mtDNA replication is strongly associated with cellular demand for ATP [33]. To investigate whether the increased MitoTracker Red fluorescence signal observed in the P49 and P07 transgenic lines is associated with alteration in mtDNA, we used the MATR maturase gene to quantify its mtDNA content relative to the WT line. qRT-PCR quantification of MATR in total DNA extracted from L1 to L5 leaves revealed an estimated mtDNA increase of 2 - to 5 -fold in the P49 and P07 transgenic lines, respectively, compared with that of WT plants (Figure 2E). Because mitochondrial biogenesis requires the coordinated expression of both nuclear and mitochondrial genomes $[17,24]$, we examined whether AtUCP1 overexpression results in the upregulation of other nuclear- and mitochondrial-encoded mitochondrial proteins. q-PCR of total RNA extracted from L1 to L5 leaves from P49, P07 and WT plants was used to quantify relative mRNA content from genes encoding a selected set of nuclear- and mitochondrial-encoded mitochondrial proteins. Independent of leaf age, the P49 and P07 transgenic lines presented 2- to 5-fold increases in the transcript abundance of $\mathrm{NADH}$ dehydrogenase (NADH-DeH) and NADH iron-sulfur proteins 2 and 7 (NADH IS2-7), which are components of mitochondrial respiratory chain complex I [34] (Figure 2F). The increases in both nuclear-encoded (NADH-DeH and NADH-IS7) and mitochondrial-encoded (NADH-IS2) components of complex I indicate that mitochondrial/nuclear coordination is necessary for bioenergetic adaptation. mIncreased transcript abundance in P49 and P07 transgenic lines was also 


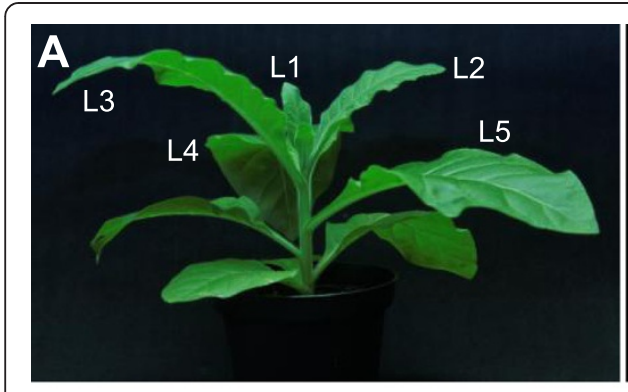

E

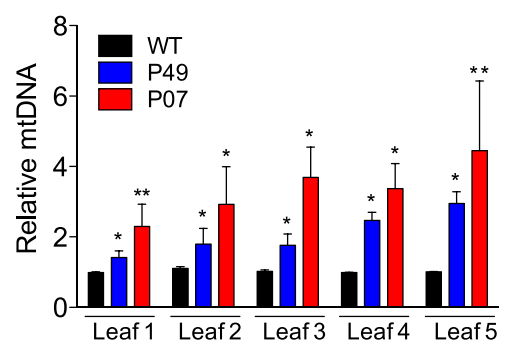

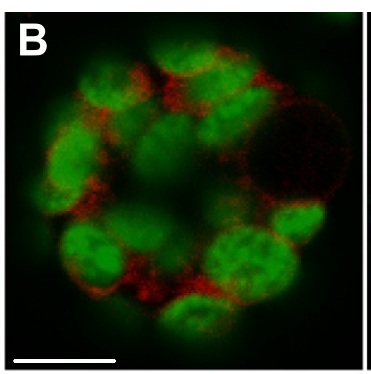

$\mathbf{F}$

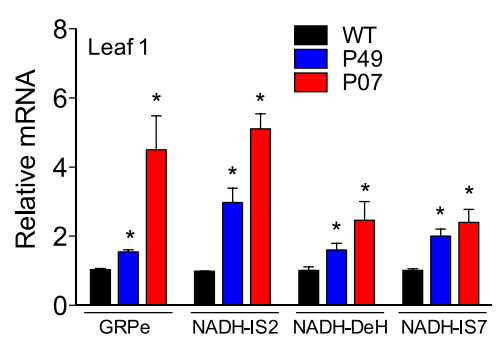

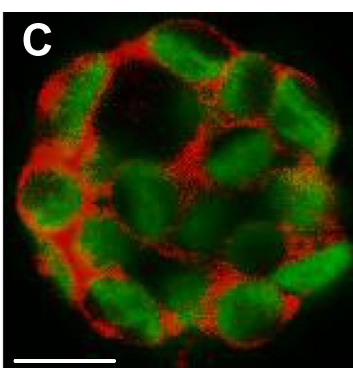

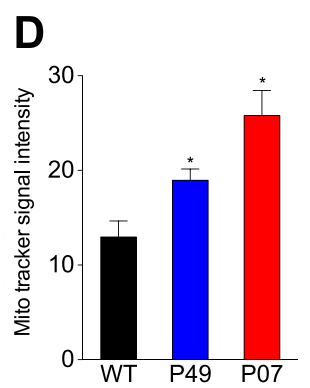

G

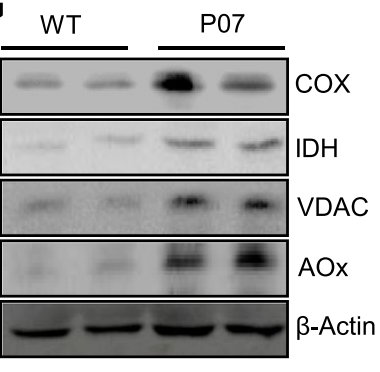

Figure 2 Overexpression of $A t U C P 1$ in tobacco induces mitochondrial biogenesis through retrograde signaling. (A) Five leaves, from youngest (L1) to oldest (L5), were sampled from WT and transgenic lines P49 and P07. (B and C) Mesophyll protoplasts isolated from WT (B) and P07 (C) L3 leaves stained with MitoTracker Red. The panels are representative of 50 protoplasts evaluated for each genotype. Scale bars $=5 \mu \mathrm{m}$. (D) Quantification of the MitoTracker Red signal in a population of protoplasts isolated from the L3 leaves of WT and transgenic lines P49 and P07 $(n=50)$. (E) Relative mtDNA content of WT and transgenic lines P49 and P07 in leaves L1 to L5. (F) Relative mRNA content of genes involved in targeting proteins to mitochondria (GRPe chaperone) and energy metabolism (NADH-DeH, NADH-IS2, and NADH-IS7) in L1 leaves of WT and transgenic lines P49 and P07. (G) Immunoblot analysis of nuclear- (IDH, VDAC and AOX) and mitochondrial-encoded (COXII) proteins involved in energy metabolism, transport, and stress response in the L1 leaves of WT and transgenic line P07. The data presented in panels $\mathbf{E}$ and $\mathbf{F}$ are representative of four biological replicates $(n=4) .{ }^{*} p<0.05$ and ${ }^{* *} p<0.1$ compared with WT. Error bars, mean \pm s.e.m.

observed for the molecular chaperone GRPe and for the adenine nucleotide exchange factor of heat shock protein70 (HSP70) that is involved in mitochondrial targeting of proteins [35] (Figure 2F and Additional file 1: Figure S1A-D). We also observed that the P07 transgenic line showed 2- to 5-fold increases in the protein levels of cytochrome oxidase subunit II (COXII), isocitrate dehydrogenase (IDH), voltage-dependent anion channel-1 (VDAC1), and alternative oxidase (AOX), which are proteins associated with both oxidative stress [36] and mitochondrial metabolism [37-39] (Figure 2G). These data indicate that AtUCP1 overexpression triggered retrograde signaling, leading to increased transcription and translation of mitochondrial proteins encoded by both the nuclear and mitochondrial genomes, culminating in increased mitochondrial biogenesis.

\section{AtUCP1 overexpression increases uncoupling respiratory capacity and decreases cellular ATP content}

Although AtUCP1 overexpression protects tobacco plants from oxidative, osmotic and drought stress, no previous data indicate whether UCP1 does, in fact, uncouple ATP synthesis in these plants [11]. However, previous work has demonstrated that mitochondria isolated from Solanum tuberosum (potato) plants overexpressing a UCP show increased uncoupled respiration [40]. Here, we demonstrate that crude mitochondria isolated from L3 leaves of WT and P07 plants retained their respiratory properties: ADP and FCCP can efficiently increase oxygen consumption when compared to basal respiration (Figure 3A). Additionally, transgenic plants showed decreased adenosine 5'-diphosphate (ADP)-dependent respiration and increased uncoupling respiration in comparison to WT plants (Figure 3B). There was no significant difference in the oxygen consumption in the presence of carbonyl cyanide-4-trifluoromethoxy phenylhydrazone (FCCP) between P07 and WT mitochondria (Figure 3B). The increased uncoupling respiration of P07 compared with WT mitochondria is evident in a comparison of the leak ratios (uncoupled/maximum oxygen consumption) in P07 and WT (Figure 3B). The increased uncoupling respiration in the P07 transgenic line resulted in $20-35 \%$ decreased cellular ATP concentration in the L3 leaves compared with the WT (Figure 3C).

Altered mitochondrial metabolism due to increased UCP1 activity leads to changes in mitochondrial number, volume and morphology

Mitochondrial fission and fusion dynamics, as well as mitochondrial morphology, have been shown to be directly 

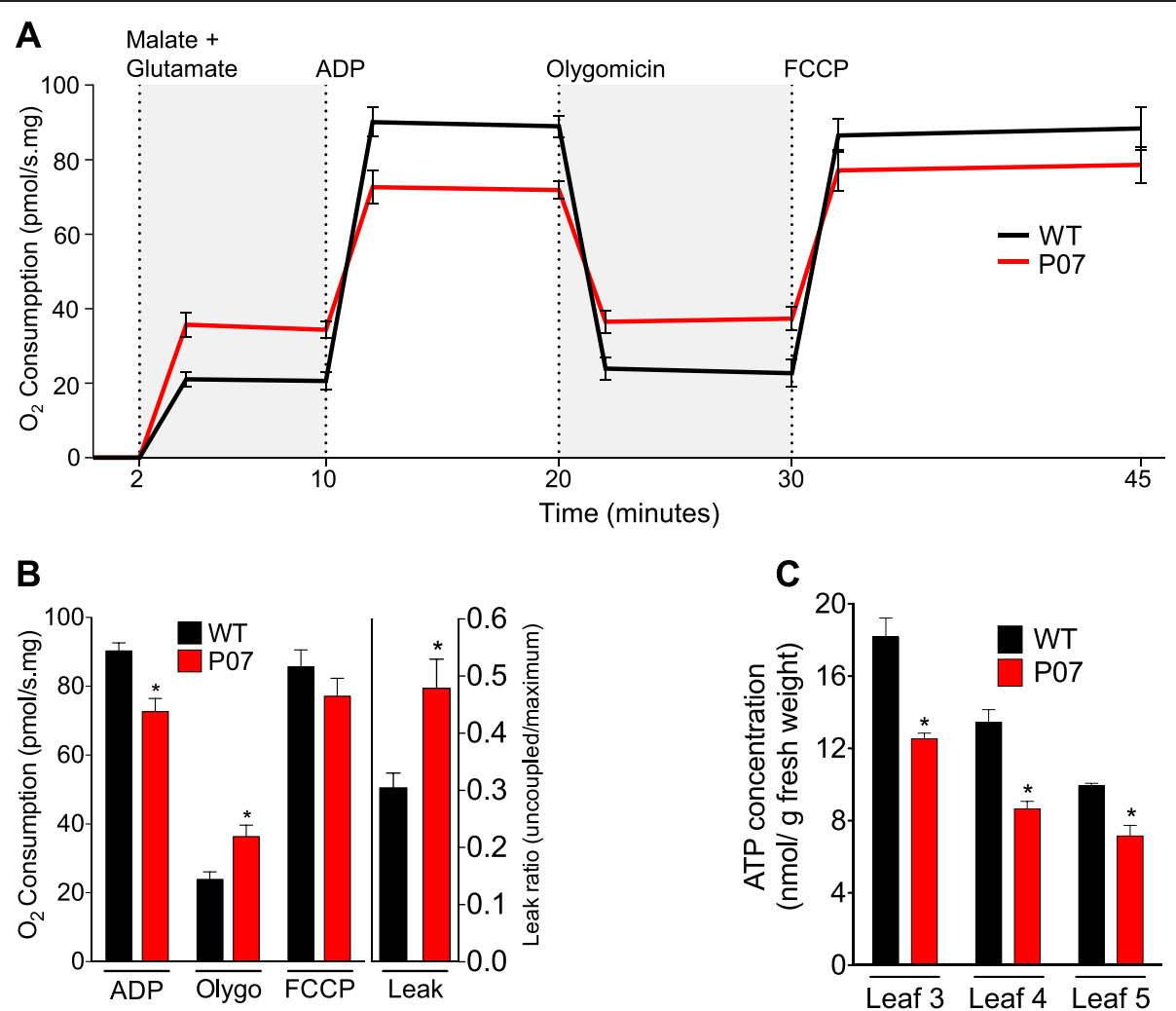

Figure 3 Overexpression of AtUCP1 increases mitochondrial uncoupled respiration, resulting in decreased cellular ATP content.

(A) Time-course of respiration measurements on isolated mitochondria from WT and P07 plants. Malate (10 mM) and glutamate (5 mM) were added to the respiration medium to provide substrates for the electron transport chain. ADP ( $800 \mu \mathrm{M})$ was used to stimulate ADP-dependent respiration, and the ATP synthase inhibitor oligomycin $(5 \mu \mathrm{M})$ was used to measure oxygen consumption due to uncoupled respiration. Maximum oxygen consumption was measured in the presence of FCCP $(2 \mu \mathrm{M})$. (B) Average oxygen consumption of WT and P07 isolated mitochondria in the presence of ADP, oligomycin and FCCP. The leak ratio was calculated as the ratio between oxygen consumption in the presence of oligomycin and that in the presence of FCCP. (C) Decreased ATP levels in the L3, L4 and L5 leaves of the P07 transgenic line compared with WT. The data are representative of six biological replicates $(n=6)$. ${ }^{*} p<0.05$ and ${ }^{* *} p<0.1$ compared with WT. Error bars, mean \pm s.e.m.

affected by modulations in the energy demand and nutrient supply in human cells [41]. The fine regulation of these parameters is linked to the adaptation of mitochondrial architecture to metabolic demand. To address whether AtUCP1 overexpression affects mitochondrial morphology, we performed transmission electron microscopy (TEM) of mesophyll cells of L2 leaves from P07 and WT plants. Approximately 20 micrograph fields for each genotype were analyzed for mitochondrial number and area using ImageJ [42]. Images from P07 leaves revealed increases of 1.6-fold in mitochondrial number and 1.4-fold in mitochondrial volume, resulting in an overall augmentation of 2.2-fold in the total mitochondrial area (Figure 4A, B, and C). Surprisingly, and perhaps for the first time, we observed a novel mitochondrial morphology in plant cells: donut-shaped mitochondria (Figure 4D). This morphology has been observed in human cells during treatment with FCCP or subjection to hypoxic stress [43]. These treatments decrease oxygen availability for ATP production. This unusual morphology has also been observed during reoxygenation and
FCCP washout, when cells reoxygenate and ATP levels are partially restored. When ATP levels were completely restored, the donut-shaped mitochondria fragmented, generating new mitochondria. These findings suggest that AtUCP1 overexpression triggers a retrograde signal that promotes mitochondrial biogenesis. The alterations in both mitochondrial architecture and morphology may be due to metabolic stress imposed by the higher activity of the electron transport chain, thus increasing oxygen consumption, together with the reduced ATP synthesis caused by the AtUCP1 overexpression. The donut-shaped mitochondrial morphology might be associated with the fusion/fission dynamics but may also be important to alleviate the tension imposed by the increased mitochondrial volume [43].

\section{AtUCP1 overexpression induces broad transcriptomic} activation of mitochondrial and stress-responsive genes It was unexpected that the overexpression of a single protein would result in mitochondrial biogenesis because the 

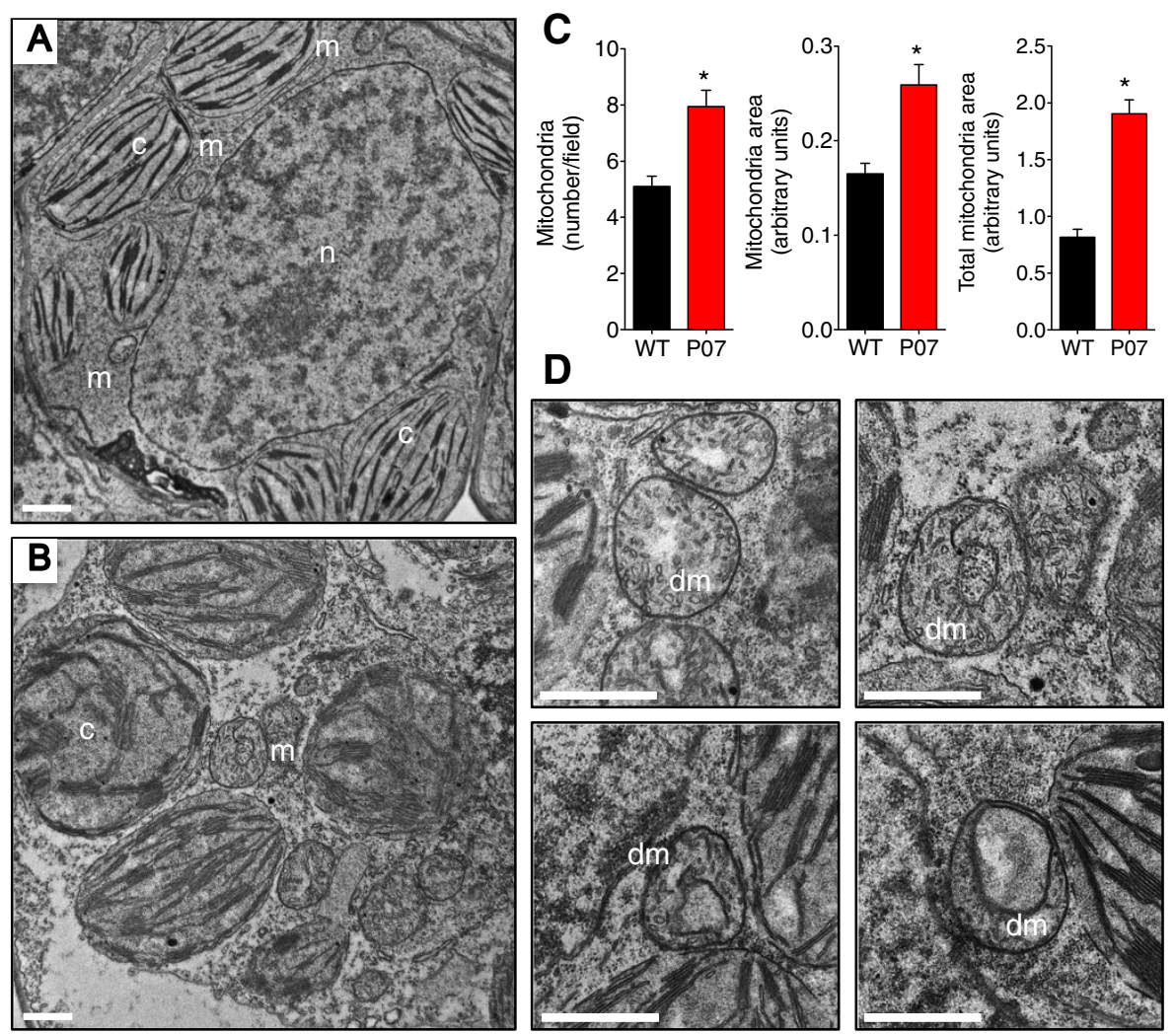

Figure 4 Overexpression of AtUCP1 increased mitochondrial biogenesis through donut formation/fragmentation. (A) Representative TEM micrograph of WT L2 leaves. (B) Representative TEM micrograph of P07 L2 leaves. (C) Quantification of the mitochondrial number, area, and total area occupied by mitochondria in TEM micrograph field images of WT and P07 plants. (D) Close-up of the donut-shaped mitochondria at different developmental stages in P07 transgenic cells. Image analysis was performed on 20 field images $(n=20)$ for each genotype. * $p<0.05$ compared with WT. Error bars, mean \pm s.e.m. Scale bars, $1 \mu \mathrm{m}$.

mitochondrial proteome is composed of over 2000 proteins [24]. Therefore, the induction of mitochondrial biogenesis would require the expression of an array of genes encoding these proteins. To examine the extent of retrograde signaling activation due to AtUCP1 overexpression, we conducted a global transcriptomic analysis of L3 leaves from P07 and WT plants. The transcripts were annotated using UniProt [44] and categorized into the Clustering of Orthologous Groups (COG) database [45]. We used TAIR subcellular prediction of proteins [46] to identify a total of 705 nuclear-encoded mitochondrial transcripts that have significantly higher RPKM values in the P07 transgenic line and 165 that have higher expression levels in WT plants (Figure 5A, red dots). In addition, we identified 12 mitochondrial-encoded proteins, all of which were more than 2-fold (significantly) upregulated in the P07 transgenic line (Figure 5A, blue dots). The most representative mitochondrial upregulated proteins include those associated with energy production, pentatricopeptide proteins, proteins associated with mitochondrial transcription and translation, and a large number of unknown proteins
(Figure 5B and Additional file 2: Table S1). Proteins with unknown subcellular prediction were submitted to further analysis using TargetP [47]. This analysis allowed us to identify another 43 proteins predicted to be targeted to mitochondria with fold-changes higher than 2.0 in P07 plants (Additional file 3: Table S2). Among several proteins with unknown function, we identified possible orthologs of one of the mitochondrial calcium uniporters (MCUs) and a mitochondrial sodium/calcium exchanger (MCX). One of these proteins (Solyc04g079910.2.1 in Additional file 3: Table S2), which is 2.5 -fold upregulated in P07, contains a coiled-coil domain on the C-terminal region, which is found among the MCUs, and has a strong sequence similarity with the Arabidopsis thaliana (AtMCU), Mus musculus (MmMCU) and Homo sapiens (HsMCU) mitochondrial calcium uniporters. The other protein (Solyc 07g042000.2.1 in Table 2) contains a sodium/calciumexchanging domain whose sequence is predicted to be targeted to mitochondria. Among the upregulated proteins in the P07 transgenic line, we also identified several proteins associated with calcium signaling, mitochondrial 


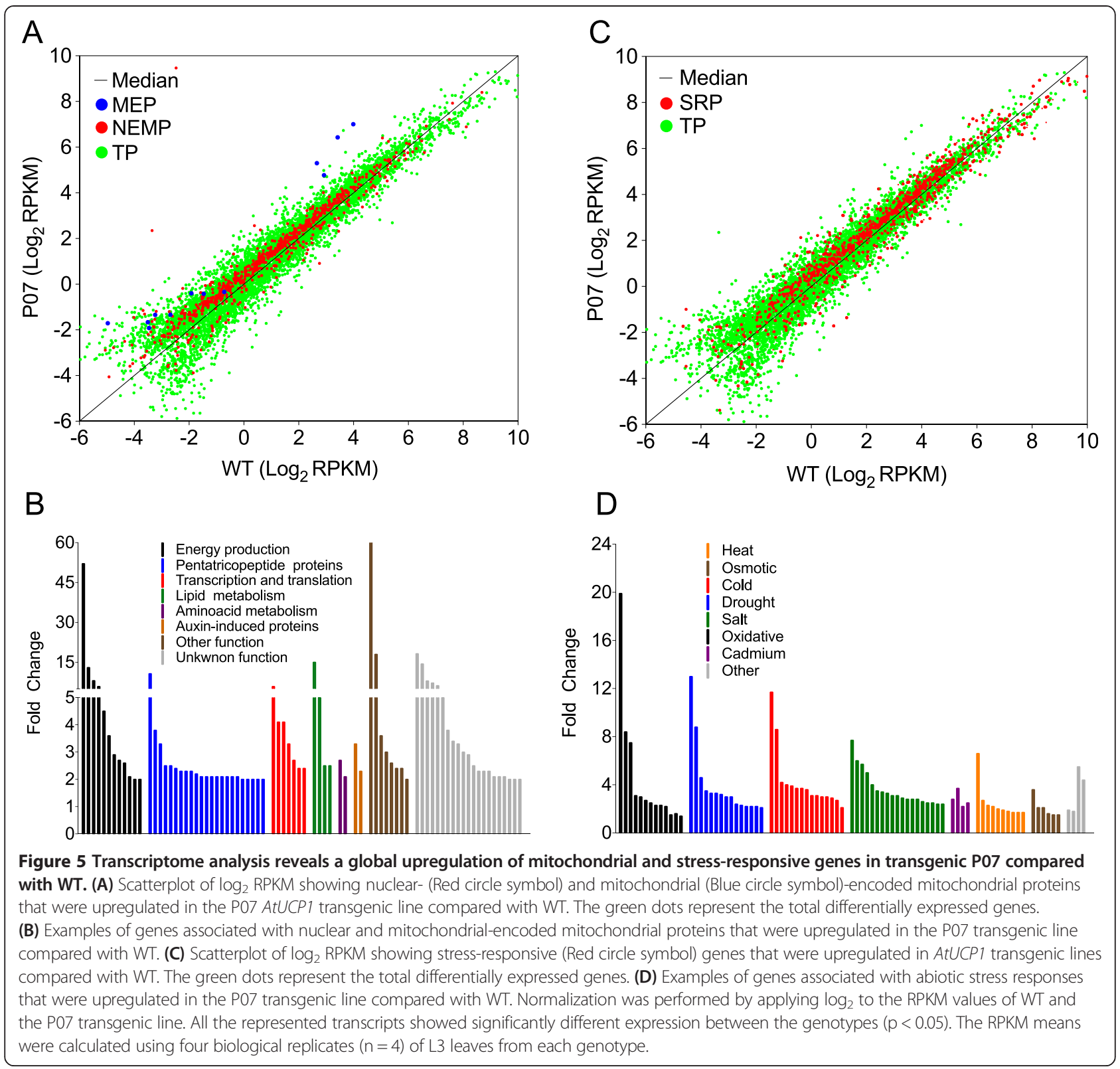

import machinery and mitochondrial fission and fusion processes, along with transcripts related to lipid metabolism, which may act to support UCP1 activity and cell metabolism (Additional file 4: Table S3). Several transcription factors that may play a role in mitochondrial retrograde signaling were also identified (Additional file 4: Table S3). Surprisingly, we also identified 1071 genes responsive to abiotic stress, $72 \%$ (770) of which have increased expression in the P07 transgenic line compared with WT (Figure 5C, red dots). Among these stress-associated proteins, we identified large numbers associated with heat, osmotic, cold, drought, salt, oxidative and cadmium stress (Figure 5D). We also observed 1.4- to 3.4-fold increases in the transcript levels of genes involved in antioxidant mechanisms, including SOD, GPX and GSTs, among the upregulated stressresponsive genes (Additional file 5: Table S4).

\section{UCP1 transgenic line performed better under abiotic stresses}

In addition to the previous observation of increased tolerance to oxidative, salt, and drought stresses [11,12], we observed that the P07 UCP1 overexpressor performed better under low and high $\mathrm{pH}$, hyperosmotic stress, and oxidative stress (Figure 6A). Additionally, in the presence of free fatty acids, the P07 transgenic line also exhibited improved performance under low temperature, a result that 


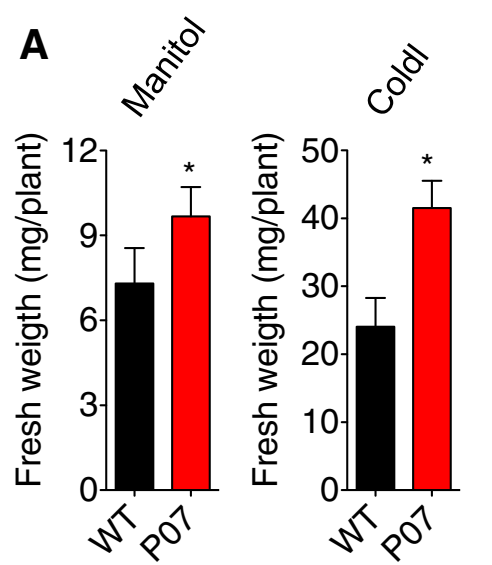

B
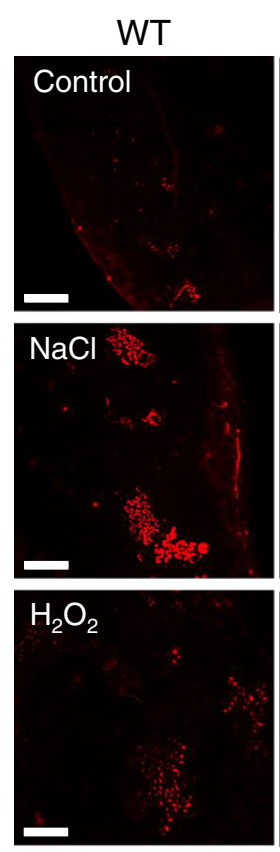
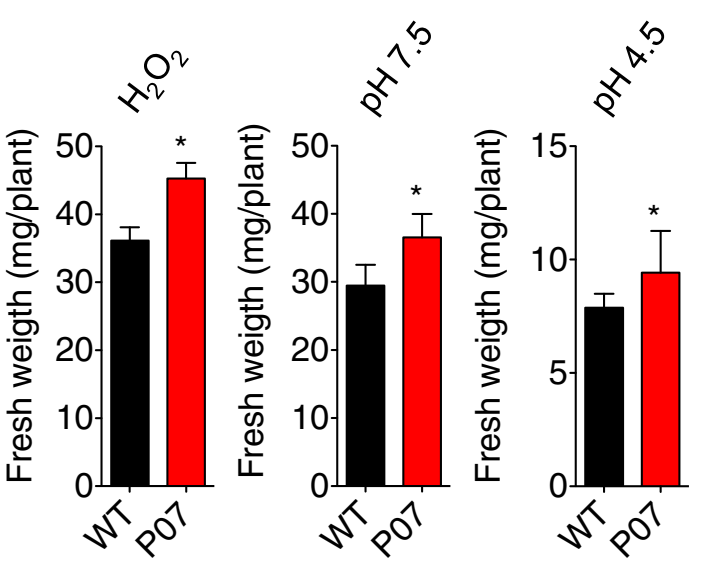

C
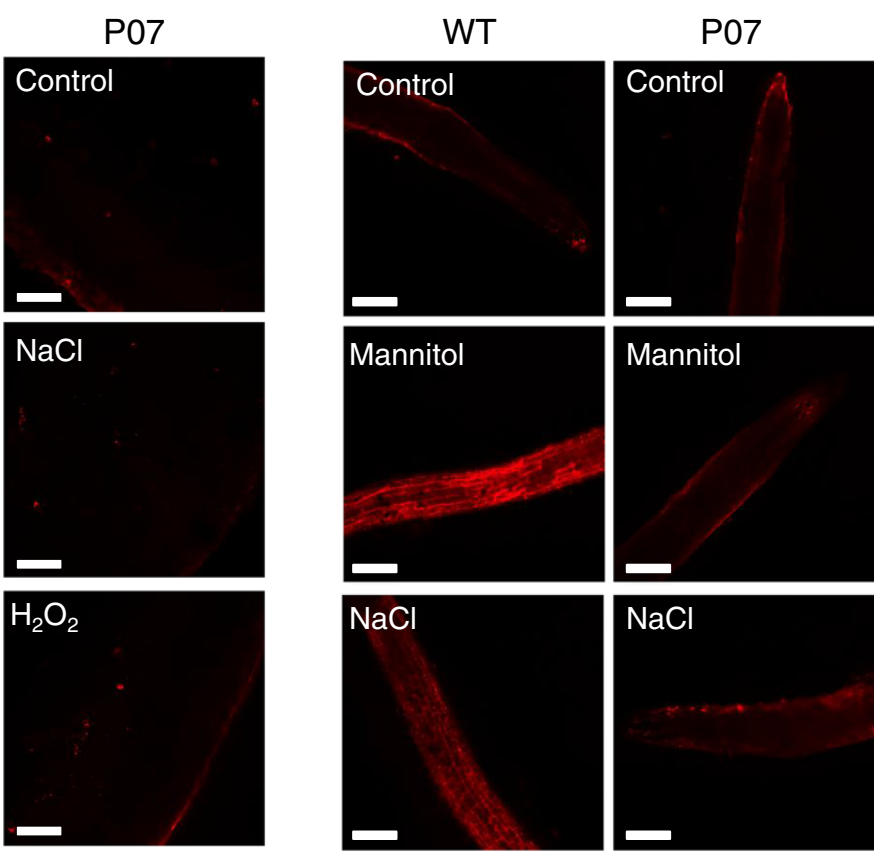

Figure 6 AtUCP1-overexpressing plants performed better under abiotic stress, most likely by decreasing ROS accumulation.

(A) Two-week-old WT and P07 plantlets were plated on half-strength MS medium supplemented with 80 mM mannitol (hyperosmotic stress) or $10 \mathrm{mM} \mathrm{H}_{2} \mathrm{O}_{2}$ (oxidative stress), $\mathrm{pH} 7.5$ and pH 4.5 for 22 days under a day/light regime of $16 \mathrm{~h}$ at $100 \mu \mathrm{E} \mathrm{m}^{-2} \mathrm{~s}^{-1}$ and $8 \mathrm{~h}$ in darkness. For the low-temperature treatment, the seeds were germinated and grown for 30 days on half-strength MS medium supplemented with $50 \mu \mathrm{M}: 10 \mu \mathrm{M}$ linoleic acid:bovine serum albumin. Plant growth assays were performed using four biological replicates $(n=4)$ on plates containing 5 plants for each treatment. ${ }^{*} p<0.05$ and ${ }^{* *} p<0.1$ compared with WT. Error bars, mean \pm s.e.m. Scale bars, $200 \mu \mathrm{m}$. (B) Leaf discs $(0.8 \mathrm{~cm})$ or (C) root tips isolated from WT and the P07 transgenic line were incubated in half-strength MS medium supplemented with $100 \mathrm{mM} \mathrm{NaCl}, 100 \mathrm{mM}$ mannitol, or $10 \mathrm{mM} \mathrm{H}_{2} \mathrm{O}_{2}$ for $20 \mathrm{~h}$. Control treatments were performed using unsupplemented half-strength MS medium. Tissues were stained with MitoSox red and visualized with fluorescent confocal microscopy.

support the thermogenic role of plant UCP1 (Figure 6A). Because UCP1 activity in mouse brown adipose tissue, potato tuber and tobacco leaves can reduce superoxide accumulation $[12,40,48]$, we examined whether this process also occurs in the P07 transgenic line but used an approach that detects ROS accumulation only within mitochondria. When subjected to salt, oxidative and hyperosmotic stress, mitochondrial ROS accumulation was decreased in the P07 transgenic line compared with WT plants in both leaf discs and roots (Figure $6 \mathrm{~B}$ and $\mathrm{C}$ ).

\section{Discussion}

The mitochondrial respiratory chain is composed of four macromolecular complexes through which free energy is conserved by coupling electron transport to the formation of a proton motive force by complexes I, III, and IV, 
which is then dissipated by F1FO-ATPase (complex V) for ATP synthesis [49]. In the BAT, electron transport is uncoupled from ATP synthesis by UCP1 for heat production [4]. However, BAT is a specialized tissue dedicated to thermogenesis regulation whose main characteristic is a dramatic increase in mitochondria number and UCP1 expression [50]. In other cell types, however, decreased ATP concentration may compromise cell metabolism [51]; therefore, cells somehow sense the cytoplasmic ATP concentration as a signal to change gene expression to quickly readjust the mitochondrial architecture and cell metabolism $[18,52]$. In this work, we show that the overexpression of UCP1 increases uncoupled respiration and decreases cellular ATP concentration. There is evidence demonstrating that mitochondria isolated from potato plants overexpressing UCP exhibit increased uncoupling respiration [40], but the present study represents the first time that the effect of UCP on intracellular ATP level has been quantified in plants. It is possible that the enhanced uncoupled respiration and its consequent negative effect on the ATP production trigger a signal to compensate for the energy metabolism and maintain ATP at safe levels. Consistent with this view is the remarkable upregulation of electron transport chain components, including NADHDeH, NADH-IS2-7, and COX, observed in the P07 overexpressor. The coordinated upregulation of the electron transport chain components encoded by the nuclear and mitochondrial genomes occurs in tissues with high metabolic demands and increased mitochondrial biogenesis $[21,50,53]$, such as flowers [22-24,54] and germinating seeds [55].

Mitochondrial biogenesis was observed using multiple approaches, including MitoTracker RED fluorescence imaging, quantitation of mtDNA and TEM. In all cases, these methods allowed us to conclude that the overexpression of AtUCP1 resulted in increased mitochondrial number and volume. To assess whether the upregulation of mitochondrial genes was restricted to the electron transport chain components, we performed global transcriptome sequencing and observed the upregulation of hundreds of genes encoding mitochondrial proteins. All of the identified transcripts encoded on the mtDNA were upregulated in transgenic plants, which is consistent with the increased mtDNA content. For instance, the ATP synthase gene encoded by the mtDNA was found to be upregulated by 8.1 -fold, and two of its nuclear subunits were increased by $2.7-$ and 3.6-fold. This upregulation was not restricted to the components involved in the oxidative phosphorylation but also included genes that participate in the tricarboxylic acid cycle (TCA). The key control point of the TCA is the enzyme IDH [56], which showed increased protein levels in P07 plants (Figure 2G). In fact, experiments performed with mitochondria isolated from potato plants demonstrated that UCP1 activity facilitates higher TCA flux by decreasing membrane potential and, consequently, ROS production [40].

Transcriptional regulation is the main regulatory factor in the expression of nuclear-encoded genes associated with respiration [21], but the vast majority of proteins targeted to the mitochondria are transported posttranslationally by tightly regulated machinery [57]. Among these proteins, HSP70 is involved in the transport from the cytoplasm to the mitochondrial outer membrane, whereas the mitochondrial TOM and TIM translocases are responsible for transport inside the organelle [58]. Interestingly, all of these proteins were upregulated in the P07 AtUCP1 overexpression line. The increased expression of components of the mitochondrial energy production machinery, along with the increased mitochondrial number of transgenic cells, may alter cellular energy homeostasis. Therefore, the mitochondria may consume more substrates in an attempt to boost ATP synthesis; for that purpose, these substrates must be supplied from other sources. This need might explain the increased photosynthetic rate exhibited by these plants under control conditions [12]. Transcriptome sequencing provides an important tool to investigate this possibility. We did not observe differential expression patterns between WT and P07 plants regarding chloroplast-targeted proteins (data not shown), but further analysis of the transcriptome might provide new insights into this issue.

The adaptation to changes in energy supply and demand influences not only mitochondrial gene expression but also mitochondrial architecture and morphology [41]. Alterations in mitochondrial morphology during starvation have already been observed in Arabidopsis thaliana cells cultured without a carbon source [24]. In human cells, treatment with the chemical uncoupler FCCP causes an increase in oxygen consumption and a decrease in ATP generation, provoking mitochondrial fragmentation and the appearance of donut-shaped mitochondria [43]. This fragmentation may occur to support an increase in respiration in an attempt to elevate ATP levels [41], whereas the formation of the donut-shaped morphology may be a component of a protective mechanism that helps to preserve the organelles from damage under conditions of metabolic stress [41,43]. When FCCP is washed out of these cells, the donut morphology persists until the ATP levels are completely restored, at which point the donuts fragment and new mitochondria arise. The mechanisms underlying the donut formation in UCP1-overexpressing cells might be explained by an analogy with human cells. Increased UCP1 activity decreases the membrane potential that is used for ATP synthesis, provoking an increase in mitochondrial number to balance the ATP levels in transgenic plants. Mitochondria are responsible for at least $90 \%$ of cell oxygen consumption [59]. We therefore expect that an increase in respiratory chain machinery 
and mitochondrial number, together with the constant leak caused by UCP1, would result in increased oxygen consumption per molecule of ATP generated. Thus, this metabolic stress caused by UCP1 overexpression appears sufficient to allow the formation of donut mitochondria in plant cells.

Communication between mitochondria and the nucleus is required for the adaptations that we observed in P07 transgenic plants. Although several important regulators of chloroplastic retrograde signaling have been extensively studied [60], relatively little is known about the regulators of mitochondrial retrograde signaling in plants. Several recently published studies suggest roles for the WRKY, bZIP, TCP, and NAC transcription factor families in activating the expression of nuclear-encoded mitochondrial proteins in plants [26-29]. These transcription factors recognize elements that are overrepresented in the regulatory region of mitochondrial genes and that are involved in several biotic and abiotic stress responses [21]. The colocalization of proteins in mitochondria and the nucleus appears to be an important topic that is very little explored in plants. For example, an Arabidopsis thaliana pentatricopeptide protein (PNM1) [25] was identified in both mitochondria and nucleus that may act to coordinate the expression between the two genomes. The increased expression of transcription factors that belong to the WRKY and TCP families, along with the upregulation of a large number of pentatricopeptide proteins in transgenic plants, presents the opportunity to further explore new candidates involved in mitochondrial retrograde signaling. The expression of most transcriptional regulators is further linked to cellular signals and environmental cues [21]. The most studied cellular signals involved in mitochondria-nucleus signaling are ATP, calcium and ROS [18]. Although the role of mitochondria in calcium metabolism has been extensively studied in mammals, little is known about calcium signaling by plant mitochondria [61]. Mitochondria in mammals are able to sequester cytoplasmic $\mathrm{Ca}^{+2}$, functioning as a transient calcium store for protective mechanisms $[18,62]$. Additionally, the $\mathrm{Ca}^{+2}$ levels inside the organelle are positively correlated with increased ATP production [63], although $\mathrm{Ca}^{+2}$ overaccumulation is linked to the induction of apoptosis in both mammals and plants [61]. Consistent with this relation, it is very interesting that we found two genes upregulated in transgenic plants that are possible orthologs, an MCU and an MCX, along with several genes involved in calcium signaling (Additional file 3: Tables S2 and Additional file 4: Table S3). The MCUs and MCXs have been extensively studied in animal cells, and their expression is tightly linked with mitochondrial calcium transport $[64,65]$.

The main focus of previous studies regarding UCP1 overexpression in plants has been ROS production [11-13,40].
Both potato and tobacco transgenic plants presented decreased superoxide production under abiotic stresses $[11,12,40]$. Additionally, tomato plants overexpressing UCP1 demonstrate not only an alteration on ROS production but also a possible role of UCP1 in regulating the cellular redox homeostasis [13]. The plants analyzed in the present study showed increased expression not only of the antioxidant defense system, including GPX, GSTs and SOD, but also of an array of stress-responsive genes. This effect may help explain the positive impact of AtUCP1 overexpression on the tolerance to multiple abiotic stresses. Consistent with previous data, we show in this work that AtUCP1 overexpression protects plants from a number of abiotic stresses, including high $\mathrm{pH}$, low $\mathrm{pH}$ and cold. The fact that transgenic tobacco performs better at low temperatures when the medium is supplemented with fatty acids reinforces the thermogenic role of UCP1 in plants. The importance of ROS in cell signaling is well known [66]; thus, the results regarding decreased mitochondrial ROS production under oxidative, osmotic and salt stresses also reinforce the importance of this molecule in UCP1-mediated retrograde signaling.

Together, these data indicate that AtUCP1-overexpressing lines suffer metabolic stress caused by the increased uncoupling respiration. Chronic overexpression of UCP1 can induce mitochondrial biogenesis in mammals [67]. Therefore, we propose that in the transgenic plants, the lower ATP level acts as a key element in the retrograde signaling, promoting a broad increase in the expression of both mitochondrial genes and stress-related genes. This pattern is reflected in the decreased ROS production and the better performance of these plants under various stresses. These findings advance our understanding of stress-tolerance mechanisms, mitochondrial biogenesis and bioenergetic adaptation in plants and therefore might assist in the implementation of biotechnological tools for the development of abiotic stress-tolerant plants. The overall regulation of stress-responsive genes indicates a link between UCP1activated mitochondrial biogenesis and the increased stress response exhibited by the transgenic plants.

\section{Conclusions}

In this work, we present strong evidence that the overexpression of UCP1 protein affects the mitochondrial dynamics at both structural and metabolic levels, leading to an increased mitochondria number and volume per cell. It could be argued that these changes are a consequence of the overpopulation of UCP1 protein at the inner mitochondrial membrane, thereby leading to abnormal mitochondrial structure and function. We interpret the results presented in this manuscript as a beneficial effect of the overexpression of UCP1 that triggers a retrograde signaling process, signaling the nuclear and mitochondrial genomes to increase the production of mitochondrial 
proteins. Consequently, more mitochondrial membrane is produced. The increase in UCP1, both because of the overproduction of the protein and the increased mitochondrial number and volume, leads to increased uncoupled respiration, decreasing the cellular ATP concentration. This change may trigger metabolic stress and enhances a strong stress response. Because it does not affect plant growth and development, this mechanism might be used to create crops better adapted to abiotic stress conditions.

\section{Methods}

\section{Plant materials and growth conditions}

Tobacco (Nicotiana tabacum) ecotype SR1 overexpressing AtUCP1 was obtained as previously described [11]. In this work, we used two independent transgenic lines, P49 and P07, which express AtUCP1 at intermediate and high levels, respectively. Transgenic and wild-type (WT) plants were grown under a light/dark cycle of $16 \mathrm{~h}$ at $100 \mu \mathrm{E} \mathrm{m}^{-2} \mathrm{~s}^{-1}$ and $8 \mathrm{~h}$ in darkness for 12 weeks on 50:50 soil/vermiculite mixtures at $24^{\circ} \mathrm{C}$. The plants were fertilized weekly with half-strength MS medium not supplemented with sucrose. For the cellular and molecular analysis, samples of the five youngest leaves (L1 to L5) of each genotype were collected, frozen in liquid nitrogen, and stored at $-80^{\circ} \mathrm{C}$. For the other experiments, seeds were grown directly in experimental medium, as described in the figure legends.

\section{qRT-PCR}

For mitochondrial DNA quantification, total DNA was isolated from WT, P49, and P07 plants using the plant DNAzol reagent (Invitrogen, Carlsbad, CA, USA) according to the manufacturer's protocol. For the mRNA expression analysis, total RNA was isolated from leaf tissue using the NucleoSpin Mini RNA/Protein Kit (Macherey-Nagel, Duren, Germany). cDNA was synthesized using the Revertaid First Strand cDNA Synthesis Kit (Fermentas, Vilnius, Lithuania) according to the manufacturer's protocol. qRTPCR was performed using an ABI PRISM 7500 (Applied Biosystems, Foster City, CA, USA) with SYBR Green (Applied Biosystems). The reactions were performed at least in triplicate for four biological replicates. The mRNA expression and mtDNA content analysis were normalized using ACTIN1 as an internal reference. Values are presented as the mtDNA content or transcript abundance of P49 and P07 relative to WT. Student's t-test was performed to determine significance $(\mathrm{p}<0.05)$. Primers based on the MATR gene were used for mitochondrial DNA quantification [68].

The following primers were designed for amplification: ACTIN1

FW: 5' -ACTGTCCACGAGGTCCGG-3'

RV: 5'-TGTCGGATCTTGCGCGGC-3'

AtUCP1

FW: 5' -TTGAGCAAGAAAATTCTTGCTG-3'
RV: 5'-AGGCGGAAGGAAAATTAGC-3'

NADH-DeH

FW: 5'-GGCTGAAGCGCGAGAAGAC-3'

RV: 5' -CAGGGCAGGCTTCTTGGC-3'

NADH-IS2

FW: 5'-GTGAAGTGGCGTGGCAAAC-3'

RV: 5' -TTGTGGATCGCGAAGGGAG-3'

NADH-IS7

FW: 5'-CGCAGTTGCGAAGCGAAAC-3'

RV: 5'-GGGCCATCGCGACAGAGA-3'

GRPe chaperone

FW: 5' -AAACCTTGGCTTGTGACCCA-3'

RV: 5'-TCATTCGGCCAGCTGAAGTT-3'

\section{Immunoblot analysis}

Total soluble proteins were extracted from WT, P49 and P07 plants by grinding frozen leaf tissue in $5 \times$ protein sample buffer (40 mM Tris, pH 7.4; $30 \mathrm{mM} \mathrm{NaCl}$; $10 \mathrm{mM} \beta$-mercaptoethanol; $0.1 \%$ Triton X-100; $5 \mathrm{mM}$ benzamidine). The proteins were separated on $12 \%$ SDSPAGE gels, transferred to nylon membranes, and blotted using the following polyclonal antibodies raised against Arabidopsis proteins (Agrisera, Vännäs, Sweden): COXII, VDAC (At3g01280), AOx1/2 (At2g22370 and At5g64210), IDH (At4g35260 and At2g27130), and ACTIN (At2g37620, At3g18780, At3g53750, At5g59370, At2g42100, At5g09810, At1g49240, At3g12110, and At3G46520). The bands were detected using an ImageQuant LAS500 (GE Healthcare, Little Chalfont, UK) with the SuperSignal West Pico chemiluminescent substrate (Thermo-Scientific, Waltham, MA, USA).

\section{Mitochondrial quantification in leaf mesophyll protoplasts by MitoTracker Red staining}

Tobacco mesophyll protoplasts were isolated from WT, P49, and P07 plants as previously described [69], with minor modifications. Leaf strips $(0.1 \mathrm{~cm}$ in length) were vacuum-infiltrated and incubated for $3 \mathrm{~h}$ in an enzyme solution (20 mM MOPS, pH 5.7; 0.4 M d-mannitol; 20 mM $\mathrm{KCl} ; 10 \mathrm{mM} \mathrm{CaCl}_{2} ; 0.1 \% \mathrm{BSA} ; 1.5 \%$ cellulase; $0.4 \%$ macerozyme). After digestion, the suspension was filtered through $75 \mu \mathrm{m}$ nylon mesh and centrifuged at $100 \times \mathrm{g}$ for $2 \mathrm{~min}$. The protoplasts were resuspended in W5 buffer $(2 \mathrm{mM}$ MOPS, pH 5.7; $154 \mathrm{mM} \mathrm{NaCl} ; 125 \mathrm{mM} \mathrm{CaCl}_{2} ; 5 \mathrm{mM}$ $\mathrm{KCl})$, incubated on ice for $30 \mathrm{~min}$, and subsequently resuspended in MMG solution (4 mM MOPS, pH 5.7; $0.4 \mathrm{M}$ mannitol; $15 \mathrm{mM} \mathrm{MgCl}_{2}$ ). The protoplasts were then stained with $250 \mathrm{nM}$ MitoTracker Red CM-H2XRos (Invitrogen) for $30 \mathrm{~min}$ in the dark and washed in MMG solution before viewing. MitoTracker Red CMH2XRos fluorescence was detected at Ex/Em of 543/ $589 \mathrm{~nm}$ and chlorophyll auto fluorescence was detected at Ex/Em 543/645 $\mathrm{nm}$ using a confocal microscope (LSM780-NLO; Zeiss, Oberkochen, Germany) equipped 
with a $40 \times$ oil-immersion objective. The image analysis was performed for 40 protoplasts per sample using ImageJ 1.44p [42]. MitoTracker Red CM-H2XRos fluorescence intensity was normalized using the chlorophyll autofluorescence intensity.

\section{Mitochondrial superoxide detection by MitoSox staining} Leaf discs $(0.8 \mathrm{~cm}$ in diameter) isolated from L3 leaves were treated with half-strength MS medium (control) or halfstrength MS medium supplemented with $100 \mathrm{mM} \mathrm{NaCl}$ (salt stress) or $10 \mathrm{mM} \mathrm{H} \mathrm{H}_{2}$ (oxidative stress) for $20 \mathrm{~h}$ under constant light and agitation. For root analysis, plants grown for 3 weeks on half-strength MS medium were transferred to petri dishes containing fresh half-strength MS medium not supplemented (control) or supplemented with $100 \mathrm{mM} \mathrm{NaCl}$ (salt stress) or $100 \mathrm{mM}$ mannitol (hyperosmotic stress) and kept under constant light for $20 \mathrm{~h}$. The leaf discs and roots were stained with $5 \mu \mathrm{M}$ MitoSox-Red (Invitrogen) for $25 \mathrm{~min}$ and washed with halfstrength MS medium before viewing. Fluorescence was detected at Ex/Em of 510/580 nm using a confocal microscope (LSM780-NLO; Zeiss) equipped with a $10 \times$ objective.

\section{Transmission electron microscopy}

Leaf strips (1-2 $\mathrm{mm}$ in length) from L2 leaves of WT and P07 plants were submerged and vacuum infiltrated for at least $2 \mathrm{~h}$ in fixative solution (2.5\% glutaraldehyde, $2.5 \%$ paraformaldehyde in $0.1 \mathrm{M}$ sodium cacodylate buffer, $\mathrm{pH}$ 7.4). The samples were washed in $0.1 \mathrm{M}$ cacodylate buffer and postfixed in a solution containing $1 \%$ osmium tetroxide $\left(\mathrm{OsO}_{4}\right)$ and $1.5 \%$ potassium ferrocyanide $\left(\mathrm{KFeCN}_{6}\right)$ for $1 \mathrm{~h}$. The samples were then washed 3 times in water and incubated in 1\% uranyl acetate in maleate buffer for $1 \mathrm{~h}$; this was followed by 3 washes in maleate buffer and subsequent dehydration in a graded ethanol series (50\%, $70 \%$, and 90\%, $10 \mathrm{~min}$ each; 100\%, $2 \times 10 \mathrm{~min}$ ). The samples were then placed in propylene oxide for $1 \mathrm{~h}$ and infiltrated in a 1:1 mixture of propylene oxide and TAAB 812 Resin mixture (Marivac Canada, St. Laurent, Canada). The samples were embedded in TAAB 812 Resin mixture and polymerized at $60^{\circ} \mathrm{C}$ for $48 \mathrm{~h}$. Ultrathin sections of approximately $90 \mathrm{~nm}$ were cut using a Reichert Ultracut-S microtome, placed onto copper grids, stained with lead citrate, and examined using a Tecnai G2 Spirit BioTWIN transmission electron microscope. The images were recorded with an AMT $2 \mathrm{k}$ CCD camera. The mitochondrial number and area were quantified on 20 field images representing each genotype at $4800 \times$ direct magnification. The image analysis was performed using ImageJ 1.44p [42].

\section{ATP measurement}

ATP assays were performed on L4 and L5 leaves isolated from four biological replicates of WT and P07 plants. ATP extraction was performed as previously described [70], with minor modifications. The leaves were ground in liquid nitrogen, and $30 \mathrm{mg}$ of leaf tissue was homogenized with $300 \mu \mathrm{L}$ of $0.1 \mathrm{M} \mathrm{HCl}$ for $5 \mathrm{~min}$. The homogenate was centrifuged at 20,000 $\times \mathrm{g}$ for $10 \mathrm{~min}$, and the supernatant was centrifuged using a microconcentrator Ultra-15 (Millipore, Billerica-USA) at $14,000 \times \mathrm{g}$ for $20 \mathrm{~min}$. The ATP content was determined by using an ATP assay kit (Invitrogen) and a GloMax luminometer (Promega, Fitchburg, WI, USA).

\section{Measurement of mitochondrial respiration}

Measurements of mitochondrial respiration were performed using crude isolated mitochondria from six biological replicates of WT and P07 plants. The isolation of crude mitochondria was performed as previously described [71], with minor modifications. All steps were performed at $4^{\circ} \mathrm{C}$. Approximately $5 \mathrm{~g}$ of leaves were cut with a razor blade and ground in $20 \mathrm{~mL}$ of grinding buffer (0.3 M sucrose, $60 \mathrm{mM}$ TES, $10 \mathrm{mM}$ EDTA, $10 \mathrm{mM}$ $\mathrm{KH}_{2} \mathrm{PO}_{4}, 25 \mathrm{mM}$ disodium pyrophosphate, $1 \mathrm{mM}$ glycine, 1\% (w/v) PVP-40, 1\% (w/v) BSA, $50 \mathrm{mM}$ sodium ascorbate, $20 \mathrm{mM}$ cysteine, $\mathrm{pH} 8.0$ ). The extract was filtered through two layers of $20 \mu \mathrm{m}$ nylon mesh and one layer of Miracloth (Millipore, Billerica, MA, USA) and centrifuged twice at $2500 \times \mathrm{g}$ for $5 \mathrm{~min}$ to remove the debris. The resulting supernatant was centrifuged at $15,000 \times \mathrm{g}$ for $15 \mathrm{~min}$, and the resulting pellet was gently resuspended in $20 \mathrm{~mL}$ of wash buffer $(0.3 \mathrm{M}$ sucrose, $10 \mathrm{mM}$ TES, $2 \mathrm{mM}$ EDTA, $10 \mathrm{mM} \mathrm{KH}{ }_{2} \mathrm{PO}_{4}, \mathrm{pH}$ 7.5) and centrifuged at $15,000 \times \mathrm{g}$ for $15 \mathrm{~min}$. The pellet containing the crude mitochondria was resuspended in $200 \mu \mathrm{L}$ of wash buffer. The respiratory measurements were performed using an Oxygraph-2 $\mathrm{k}$ respirometer (Oroboros, Innsbruck, Austria) at $25^{\circ} \mathrm{C}$ in $2 \mathrm{~mL}$ of mitochondria-containing assay buffer $(0.3 \mathrm{M}$ sucrose, $10 \mathrm{mM}$ TES, $10 \mathrm{mM} \mathrm{KCl,} 2 \mathrm{mM} \mathrm{MgSO}, 5 \mathrm{mM} \mathrm{KH}_{2} \mathrm{PO}_{4}$, $0.1 \%(\mathrm{w} / \mathrm{v})$ BSA, $2 \mu \mathrm{M}$ EGTA, pH 7.5). Malate (5 mM) and glutamate $(10 \mathrm{mM})$ were used as substrates to stimulate respiration. ADP $(800 \mu \mathrm{M})$ was added to measure ADPdependent respiration. Oligomycin $(5 \mu \mathrm{M})$ was used as an F1FO ATP synthase inhibitor to measure oxygen consumption due to uncoupling activity, and the chemical ionophore carbonyl cyanide-4-(trifluoromethoxy)phenylhydrazone (FCCP) $(2 \mu \mathrm{M})$ was used to determine the maximum oxygen consumption.

\section{Transcriptome sequencing, assembly, and mapping}

Total RNA isolated from four biological replicates of WT and P07 L3 leaves was used to create single-end RNASeq libraries using the Illumina TruSeq RNA Sample Prep Kit (Illumina, San Diego, CA, USA) according to the manufacturer's instructions. The libraries were sequenced in four lanes of an Illumina HiSeq 2000 for 75 cycles. A total of $42.7 \mathrm{~Gb}$ of sequence was generated for the 8 libraries, with a minimum of 27.9 million 
and a maximum of 113.2 million reads for each library (Additional file 6: Table S5). The Illumina reads were filtered to remove adapters and low-quality reads (reads $<70 \%$ bases with quality $\geq$ Q20) using AdapterRemoval, the FASTX-Toolkit (http://hannonlab.cshl. edu/fastx_toolkit), and Perl scripts. After the filtering step, the reads were subjected to digital normalization using the diginorm software (https://github.com/ged-lab/ 2012-paper-diginorm). The resulting 49.5 million reads were assembled with the Trinity [72] assembler, generating 271,750 transcript contigs. High-quality reads (569 million reads) were mapped to Trinity contigs using Bowtie [73], and the RPKM [74] values were calculated. Because the $N$. tabacum genome is not yet completely sequenced and the scaffolds of $N$. benthamiana are estimated to cover only $79 \%$ of its genome, we used the complete genome sequence of Solanum lycopersicum [75], a close relative of $N$. tabacum, as a template. To identify proteincoding genes, all 271,750 contigs were used as queries in BLASTn and BLASTx searches against both the nonredundant set of 34,727 tomato-coding sequences (CDSs) and predicted protein sequences. The 271,750 contigs were compared to the Solgenomics tomato genome predicted protein database using BLASTx $\left(E-v a l u e ~<1 \mathrm{e}^{-5}\right)$. Of the total contigs, 134,752 mapped to the tomato genome, resulting in the identification of 20,045 distinct orthologs for the tomato CDS models. As representative contigs, we chose the three best matching contigs with higher average RPKM values (P07 and WT) for each tomato CDS. An Ftest was used to calculate whether the samples had equal or unequal variances, and the appropriate Student t-test was then applied to calculate the significant difference between the average RPKM of P07 versus WT in a contig-wise manner. The contigs identified as differentially expressed were selected for the next steps. The fold change value for the representative transcript was calculated as the ratio between the average RPKM values in P07 and WT. The subcellular annotation of the defined contigs was determined by BLASTx queries against the TAIR [46] protein database (release 10) and TargetP [47]. Gene function data were obtained from the existing annotations of the tomato genome. The tomato protein dataset was annotated against UniProt [44] and mapped against the COG (Eukaryotic Clusters of Orthologous Groups) [45] database with BLASTp, and COG functional categories were assigned only if the two best hits had the same COG function. Stress-related genes were mapped using BLASTx against the tomato proteome in the Stress Responsive Transcription Factor Database (STIFDB) [76], and annotations were assigned to the corresponding representative tobacco contig.

\section{Accession numbers}

Sequence data from this article can be found in the GenBank/EMBL database under the following accession numbers: AtUCP1 (NM_115271.4), Actin1 (EU938079.1), NADH-DeH (Y09109.1), NADH-IS2 (M77225.1), NADHIS7 (L16810.1), GRPe chaperone (AF098636.1), AtMCU (NP_119075), MmMCU (XP_006513531), HsMCU (NP_ 612366), AtCCX3 (NP_566474).

Transcriptome sequencing data are available from Sequence Read Archive (SRA), which is accessible through NCBI BioProject ID: PRJNA211804 under the experiment ID SUB287723.

\section{Additional files}

Additional file 1: Figure S1. Tobacco plants overexpressing AtUCP1 show an increased expression of nuclear-encoded mitochondrial genes.

Additional file 2: Table S1. Classification of mitochondrial genes upregulated in P07 compared to WT. The classification was based on a TAIR annotation of protein subcellular prediction.

Additional file 3: Table S2. Predicted mitochondrial genes upregulated with $\geq 2$-fold change in P07 compared with WT. The prediction was based on TargetP.

Additional file 4: Table S3. Classification of selected genes involved in different metabolic processes and significantly upregulated in P07 compared with WT. The classification was based on COG.

Additional file 5: Table S4. Abiotic stress-responsive genes upregulated in P07 compared with WT. The classification was based on STIFDB and shows the most upregulated genes involved in different types of abiotic stresses.

Additional file 6: Table S5. Summary of RNASeq data filtered and mapped with Bowtie.

\section{Competing interests}

The authors declare that they have no competing interests.

\section{Authors' contributions}

P.B. and P.A. designed the experiments, analyzed the data, and wrote the paper. P.B. performed the experiments. V.O. and I.A.P.N. performed the bioinformatics analysis of the RNASeq data. I.G.M. developed the transgenic plants. All authors read and approved the final manuscript.

\section{Acknowledgments}

We are grateful to Ana Paula Arruda for her valuable help with transmission electron microscopy, Anibal Vercesi and Paolo la Guardia for their assistance with the respiration assays, and Eduardo Kyota for his help with the immunoblotting experiments. We also thank the staff of the National Institute of Photonics Applied to Cell Biology (INFABIC) of the State University of Campinas (UNICAMP) for their assistance with the confocal microscopy analysis and the staff of the Life Sciences Core Facility (LaCTAD) of the State University of Campinas (UNICAMP) for their assistance with the genomic and bioinformatic analyses. Pedro Barreto was supported by an FAPESP PhD fellowship (12/00126-1). Izabella Neshich was supported by an FAPESP PhD fellowship (12/00235-5). Vagner Okura is a bioinformatician at the Life Sciences Core Facility (LaCTAD) of the State University of Campinas (UNICAMP). Paulo Arruda and Ivan G. Maia are CNPq productivity research fellows.

\section{Author details}

Centro de Biologia Molecular e Engenharia Genética, Universidade Estadual de Campinas (UNICAMP), 13083-875 Campinas, SP, Brazil. ${ }^{2}$ Departamento de Genética e Evolução, Instituto de Biologia, Universidade Estadual de Campinas (UNICAMP), 13083-875 Campinas, SP, Brazil. ${ }^{3}$ Departamento de Genética, Instituto de Biociências, UNESP, 18618-970 Botucatu, SP, Brazil.

Received: 26 February 2014 Accepted: 23 May 2014

Published: 28 May 2014 
References

1. Nedergaard J, Ricquier D, Kozak LP: Uncoupling proteins: current status and therapeutic prospects. EMBO Rep 2005, 6:917-921

2. Krauss $S$, Zhang CY, Lowell BB: The mitochondrial uncoupling-protein homologues. Nat Rev Mol Cell Biol 2005, 6:248-261

3. Andrews ZB, Diano S, Horvath TL: Mitochondrial uncoupling proteins in the CNS: in support of function and survival. Nat Rev Neurosci 2005, 6:829-840.

4. Kajimura S, Seale P, Spiegelman BM: Transcriptional control of brown fat development. Cell Metab 2010, 11:257-262.

5. Vercesi AE, Martins LS, Silva MAP, Leite HMF, Cuccovia IM, Chaimovich $\mathrm{H}$ : PUMPing plants. Nature 1995, 375:24

6. Maia IG, Benedetti CE, Leite A, Turcinelli SR, Vercesi AE, Arruda P: AtPUMP: an Arabidopsis gene encoding a plant uncoupling mitochondrial protein. FEBS Lett 1998, 429:403-406.

7. Borecky J, Nogueira FTS, Oliveira KAP, Maia IG, Vercesi AE, Arruda P: The plant energy-dissipating mitochondrial systems: depicting the genomic structure and the expression profiles of the gene families of uncoupling protein and alternative oxidase in monocots and dicots. J Exp Bot 2006, 57:849-864.

8. Vercesi AE, Borecký J, Maia IG, Arruda P, Cuccovia IM, Chaimovich H: Plant uncoupling mitochondrial proteins. Annu Rev Plant Biol 2006, 57:383-404

9. Ito-Inaba $Y$, Hida Y, Mora H, Inaba T: Molecular identity of uncoupling proteins in thermogenic skunk cabbage. Plant Cell Physiol 2008, 49:1911-1916.

10. Jezek P, Borecký J, Zácková M, Costa AD, Arruda P: Possible basic and specific functions of plant mitochondrial uncoupling protein (pUCP). Biosci Rep 2001, 21:237-245.

11. Brandalise M, Maia IG, Borecký J, Vercesi AE, Arruda P: Overexpression of plant mitochondrial uncoupling protein in transgenic tobacco increases tolerance to oxidative stress. J Bioenerg Biomembr 2003, 35:205-209.

12. Begcy K, Mariano ED, Mattiello L, Nunes AV, Mazzafera P, Maia IG, Menossi M: An Arabidopsis mitochondrial uncoupling protein confers tolerance to draught and salt stress in transgenic tobacco plants. Plos One 2011, 6:e23776.

13. Chen S, Liu A, Zhang S, Li C, Chang R, Liu D, Ahammed GJ, Lin X: Overexpression of mitochondrial uncoupling protein conferred resistance to heat stress an Botrytis cinerea infection in tomato. Plant Physiol Biochem 2013, 73:245-253.

14. Sweetlove LJ, Lytovchenko A, Morgan M, Nunes-Nesi A, Taylor NL, Baxter CJ, Eickmeier A, Fernie AR: Mitochondrial uncoupling protein is required for efficient photosynthesis. Proc Natl Acad Sci U S A 2006, 103:19587-19592.

15. Rhoads DM, Umbach AL, Subbaiah CC, Siedow JN: Mitochondrial reactive oxygen species: contribution to oxidative stress and interorganellar signaling. Plant Physiol 2006, 141:357-366.

16. Rhoads DM, Subbaiah CC: Mitochondrial retrograde regulation in plants. Mitochondrion 2007, 7:177-194.

17. Woodson JD, Chory J: Coordination of gene expression between organellar and nuclear genomes. Nat Rev Genet 2008, 9:383-395.

18. Ryan MT, Hoogenrad MJ: Mitochondrial-nuclear communications. Annu Rev Biochem 2007, 76:701-722.

19. Pesaresi P, Schneider A, Kleine T, Leister D: Interorganellar communication. Curr Opin Plant Biol 2007, 10:600-606

20. Jung HS, Chory J: Signaling between chloroplasts and the nucleus: can a systems biology approach bring clarity to a complex and highly regulated pathway. Plant Physiol 2009, 152:453-459.

21. Welchen E, Garcia L, Mansilla N, Gonzalez DH: Coordination of plant mitochondrial biogenesis: keeping pace with cellular requirements. Front Plant Sci 2013, 4:551.

22. Zabaleta E, Heiser V, Grohmann L, Brennicke A: Promoters of nuclear-encoded respiratory chain complex I genes from Arabidopsis thaliana contain a region essential for anther/pollen-specific expression. Plant J 1998, 156:49-59.

23. Binder S, Brennicke A: Gene expression in plant mitochondria: transcriptional and post-transcriptional control. Philos Trans R Soc Lond B Biol Sci 2003, 3586:181-188.

24. Giegè $P$, Sweetlove $L$, Congat $V$, Leaver $C$ J: Coordination of nuclear and mitochondrial genome expression during mitochondrial biogenesis in Arabidopsis. Plant Cell 2005, 17:1497-1512.

25. Hammani K, Gobert A, Heibieh K, Choulier L, Small L, Giegè P: An Arabidopsis dual-localized pentatricopeptide repeat protein interacts with nuclear proteins involved in gene expression regulation. Plant Cell 2011, 23:730-740

26. Roschzttardtz H, Fuentes I, Vásquez M, Corvalán C, León G, Gómez I, Araya A, Holuique L, Vicente-Carbajosa J, Jordana X: A nuclear gene encoding the iron-sulfur subunit of mitochondrial complex II is regulated by B3 domain transcription factors during seed development in Arabidopsis thaliana. Plant Physiol 2009, 150:84-95.

27. Van Aken O, Zhang B, Law S, Narsai R, Whelan J: AtWKRY40 and AtWRKY63 modulate the expression of stress-responsive nuclear genes encoding mitochondrial and chloroplast proteins. Plant Physiol 2013, 162:254-271.

28. Giraud E, Ng S, Carrie C, Duncan O, Low J, Lee CP, Van Aken O, Millar AH, Murcha M, Whelan J: TCP transcription factors link the regulation of genes encoding mitochondrial proteins with the circadian clock in Arabidopsis thaliana. Plant Cell 2010, 22:3921-3934.

29. De Clercq I, Vermeirssen V, Van Aken O, Vandepoele K, Murcha MW, Law SR, Inzé A, Ng S, Ivanova A, Rombaut D, Van de Cotte B, Jaspers P, Van de Peer Y Kangasjärvi J, Whelan J, Van Breusegem F: The membrane-bound NAC transcription factor ANAC013 functions in mitochondrial retrograde regulation of the oxidative stress response in Arabidopsis. Plant Cell 2013, 25:3472-3490

30. Ölçer H, Lloyd JC, Raines CA: Photosynthetic capacity is differentially affected by reductions in sedoheptulose-1,7-biphosphatase activity during leaf development in transgenic tobacco plants. Plant Physiol 2001, 125:982-989.

31. Rogalski M, Schöttler MA, Thiele W, Schulze WX, Bock R: Rpl33, a nonessential plastid-encoded ribosomal protein in tobacco, is required under cold stress conditions. Plant Cell 2008, 20:2221-2237.

32. Unseld M, Marienfeld JR, Brandt $P$, Brennicke $A$ : The mitochondrial genome of Arabidopsis thaliana contains 57 genes in 366,924 nucleotide. Nat Genet 1997, 15:57-61.

33. Dickinson A, Yeung KY, Donoghue J, Baker MJ, Kelly RDW, McKenzie M, Jhons TJ, St John JC: The regulation of mitochondrial DNA copy number in glioblastoma cells. Cell Death Differ 2013, 20:1644-1653.

34. Klodmann J, Senkler M, Rode C, Braun HP: Defining the protein complex proteome of plant mitochondria. Plant Physiol 2011, 157:587-598.

35. Padimam M, Reddy VS, Beachy RN, Fauquet CM: Molecular characterization of a plant mitochondrial chaperone GrpE. Plant Mol Biol 1999, 39:871-881.

36. Fiorani F, Umbach AL, Siedow JN: The alternative oxidase of plant mitochondria is involved in the acclimation of shoot growth at low temperature. A study of Arabidopsis AOX1a transgenic plants. Plant Physiol 2005, 139:1795-1805.

37. Smolková K, Jezek P: The role of mitochondrial NADPH-dependent isocitrate dehydrogenase in cancer cells. Int J Cell Biol 2012, 212:1-12.

38. Robert N, D'Erfurth I, Marmagne A, Erhardt M, Allot M, Boivin K, Gissot L, Monachello D, Michaud M, Duchêne AM, Barbier-Brygoo H, Maréchal-Drouard L, Ephritikhine G, Filleur S: Voltage-dependent-anionchannels (VDACs) in Arabidopsis have a dual localization in the cell but show a distinct role in mitochondria. Plant Mol Biol 2012, 78:431-446.

39. Leadsham JE, Sanders G, Giannaki S, Bastow EL, Hutton R, Naeimi WR, Breitenbach M, Gourlay CW: Loss of cytochrome c oxidase promotes RAS-dependent ROS production from the ER resident NADPH oxidase, Yno1p, in yeast. Cell Metab 2013, 18:279-286.

40. Smith $A M O$, Ratcliffe $G$, Sweetlove L: Activation and function of mitochondrial uncoupling protein in plants. J Biol Chem 2004, 279:51944-51952

41. Liesa M, Shirihai OS: Mitochondrial dynamics in the regulation of nutrient utilization and energy expenditure. Cell Metab 2013, 17:491-506.

42. Schneider CA, Rasband WS, Eliceir KW: NIH image to ImageJ: 25 years of image analysis. Nat Methods 2012, 9:671-675.

43. Liu X, Hajnóczky G: Altered fusion dynamics underlie unique morphological changes in mitochondria during hypoxia-reoxygenation stress. Cell Death Differ 2011, 18:1561-1572.

44. The UniProt Consortium: Update on activities at the universal protein resource (UniProt) in 2013. Nucleic Acids Res 2013, 41:D43-D47.

45. Tatusov RL, Fedorova ND, Jackson JD, Jacobs AR, Kiryutin B, Koonin EV, Krylov DM, Mazumder R, Mekhedov SL, Nikoslkaya AN, Rao BS, Smirnov AS, Sverdlove AV, Vasudevan S, Wolf YI, Yin JJ, Natale DA: The COG database: an updated version includes eukaryotes. BMC Bioinforma 2003, 4:41.

46. Lamesch P, Berardini TZ, Li D, Swarbreck D, Wilks C, Sasidharan R, Muller R, Dreher K, Alexander DL, Garcia-Hernandez M, Karthikeyan AS, Lee CH, Nelson WD, Ploetz L, Singh S, Wensel A, Huala E: The Arabidopsis 
information resource (TAIR): improved gene annotation and new tools. Nucleic Acids Res 2012, 40:1202-1210.

47. Emanuelsson O, Brunak S, Heijne VJ, Nielsen H: Locating proteins in the cell using TargetP, SignalP, and related tools. Nat Protoc 2007, 2:953-971.

48. Oelkrug R, Kutschke M, Meyer CW, Heldmaier G, Jastroch M: Uncoupling protein 1 decreases superoxide production in brown adipose tissue mitochondria. J Biol Chem 2010, 285:21961-21968.

49. Vafai SB, Mootha VK: Mitochondrial disorders as windows into an ancient organelle. Nature 2012, 491:374-383.

50. Wu Z, Puigserver P, Andersson U, Zhang C, Adelmant G, Mootha V, Troy A Cinti S, Lowell B, Scarpulla RC, Spiegelman BM: Mechanisms controlling mitochondrial biogenesis and respiration through the thermogenic coactivator PGC-1. Cell 1999, 98:115-124.

51. Miyoshi N, Oubrahim H, Chock PB, Stadtman ER: Age-dependent cell death and the role of ATP in hydrogen-peroxide-induced apoptosis and necrosis. Proc Natl Acad Sci U S A 2006, 103:1727-1731.

52. Moghadam AA, Ebrahimie E, Taghavi SM, Niazi A, Babgohari MZ, Deihimi T, Djavaheri M, Ramezani A: How the nucleus and mitochondria communicate in energy production during stress: nuclear MtATP6, an early-stress responsive gene, regulates the mitochondrial $\mathrm{F}_{1} \mathrm{~F}_{0}-\mathrm{ATP}$ synthase complex. Mol Biotechnol 2013, 54:756-769.

53. Lin J, Wu H, Tarr PT, Zhang CY, Wu Z, Boss O, Michael LF, Puigserver P, Isotani E, Olson EN, Lowell BB, Bassel-Duby R, Spiegelman BM: Transcriptional co-activator PGC-1 alpha drives the formation of slow twitch muscle fibres. Nature 2002, 418:797-801

54. Peters K, Niessen M, Peterhansel C, Spath B, Holzle A, Binder S, Marchfelder A, Braun HP: Complex I-complex II ratio strongly differs in various organs of Arabidopsis thaliana. Plant Mol Biol 2012, 79:273-284.

55. Law SR, Narsai R, Taylor NL, Dellanoy E, Carrie C, Giraud E, Millar AH, Small I, Whelan J: Nucleotide and RNA metabolism prime translational initiation in the earliest events of mitochondrial biogenesis during Arabidopsis germination. Plant Physiol 2012, 158:1610-1627.

56. Berg JM, Tymoczko JL, Stryer L: Biochemistry. In The Citric Acid Cycle. 5th edition. New York: W H Freeman; 2002.

57. Pfanner N, Geissler A: Versatility of the mitochondrial protein import machinery. Nat Rev Mol Cell Biol 2001, 2:339-349.

58. Schleiffe E, Becker T: Common ground for protein translocation: access control for mitochondria and chloroplasts. Nat Rev Mol Cell Biol 2011, 12:48-59.

59. Searcy DG: Metabolic integration during the evolutionary origin of mitochondria. Cell Res 2003, 13:229-238

60. Kleine T, Leister D: Retrograde signals galore. Front Plant Sci 2013, 4:1-3.

61. Stael S, Wurzinger B, Mair A, Mehlmer N, Vothknecht UC, Teige M: Plant organellar calcium signaling: an emerging field. J Exp Bot 2012, 63:1525-1542

62. Clapham DE: Calcium signaling. Cell 2007, 131:1047-1058.

63. Tarasov Al, Griffiths EJ, Rutter GA: Regulation of ATP production by mitochondrial $\mathrm{Ca}+2$. Cell Calcium 2012, 52:28-35.

64. Alam MR, Groschner LN, Parichatikanond W, Kuo L, Bondarenko Al, Rost R, Waldeck-Weiermaier M, Mali R, Graier WF: Mitochondrial Ca2+ uptake 1 (MICU1) and mitochondrial $\mathrm{Ca}+2$ uniporter (MCU) contribute to metabolism secretion coupling in clonal pancreatic $\beta$-cells. J Biol Chem 2012, 287:34445-34454.

65. Palty R, Silverman WF, Herschfinkel M, Caporale T, Sensi SL, Parnis J, Nolte C, Fishman D, Shoshan-Barmatz D, Herrmann S, Khananshvili D, Sekler I: NCLX is an essential component of mitochondrial $\mathrm{Na}^{+} / \mathrm{Ca}^{+2}$ exchange. Proc Natl Acad Sci U S A 2010, 107:436-441.

66. Mailloux RJ, Harper ME: Mitochondrial proticity and ROS signaling: lessons from the uncoupling proteins. Trends Endocrinol Metab 2012, 23:451-458.

67. Si Y, Palani S, Jayaraman A, Lee K: Effects of forced uncoupling protein 1 expression in 3T3-L1 cells on mitochondrial function and lipid metabolism. J Lipid Res 2007, 48:826-836.

68. Wang DY, Zhang Q, Liu Y, Lin ZF, Zhang SX, Sun MX, Sodmergen: The levels of male gametic mitochondrial DNA are highly regulated in angiosperms with regard to mitochondrial inheritance. Plant Cell 2010, 22:2402-2416

69. Yoo SD, Cho YH, Sheen J: Arabidopsis mesophyll protoplasts: a versatile cell system for transient gene expression analysis. Nat Protoc 2007, 2:1565-1572.

70. Ogawa K, Hatano-Iwasaki A, Yanagida M, Iwabuchi M: Level of glutathione is regulated by ATP-dependent ligation of glutamate and cysteine through photosynthesis in Arabidopsis thaliana: mechanism of strong interaction of light intensity with flowering. Plant Cell Physiol 2004, 45:1-8.

71. Keech $\mathrm{O}$, Dizengremel P, Gardestrom P: Preparation of leaf mitochondria from Arabidopsis thaliana. Physiol Plant 2005, 124:403-409.

72. Grabherr MG, Haas BJ, Yassour M, Levin JZ, Thompson DA, Amit I, Adiconis X, Fan L, Raychowdhury R, Zeng Q, Chen Z, Mauceli E, Hacohen N, Gnirke A Rhind N, di Palma F, Birren BW, Nusbaum C, Lindblad-Toh K, Friedman N, Regev A: Full-length transcriptome assembly from RNA-seq data without reference genome. Nat Biotechnol 2011, 29:644-652.

73. Wagner GP, Kin K, Lynch VJ: Measurement of mRNA abundance using RNA-seq data: RPKM measure is inconsistent among samples. Theory Biosci 2012, 131:281-285.

74. Langmead B, Trapnell C, Pop M, Salzberg SL: Ultrafast and memory-efficient alignment of short DNA sequences to the human genome. Genome Biol 2009, 10:R25.

75. Tomato Genome Consortium: The tomato genome sequence provides insights into fleshy fruit evolution. Nature 2012, 485:635-641.

76. Naika M, Shameer K, Mathew OK, Gowda R, Sowdhamini R: STIFDB2: An updated version of plant stress-responsive transcription factor database with additional stress signals, stress-responsive transcription factor binding sites and stress-responsive genes in Arabidopsis and rice. Plant Cell Physiol 2013, 54:1-15.

doi:10.1186/1471-2229-14-144

Cite this article as: Barreto et al: Overexpression of UCP1 in tobacco induces mitochondrial biogenesis and amplifies a broad stress response. BMC Plant Biology 2014 14:144.

\section{Submit your next manuscript to BioMed Central and take full advantage of:}

- Convenient online submission

- Thorough peer review

- No space constraints or color figure charges

- Immediate publication on acceptance

- Inclusion in PubMed, CAS, Scopus and Google Scholar

- Research which is freely available for redistribution 